\title{
Magnetic field intensification: comparison of 3D MHD simulations with Hinode/SP results
}

\author{
S. Danilovic ${ }^{1,2}$, M. Schüssler ${ }^{1}$, and S. K. Solanki ${ }^{1,3}$ \\ 1 Max-Planck-Institut für Sonnensystemforschung, Max-Planck-Straße 2, 37191 Katlenburg-Lindau, Germany \\ e-mail: danilovic@mps.mpg.de \\ 2 Astronomical Observatory, Volgina 7, 11160 Belgrade 74, Serbia \\ 3 School of Space Research, Kyung Hee University, Yongin, Gyeonggi, 446-701, Korea
}

Received 6 April 2009 / Accepted 9 October 2009

ABSTRACT

\begin{abstract}
Context. Recent spectro-polarimetric observations have provided detailed measurements of magnetic field, velocity and intensity during events of magnetic field intensification in the solar photosphere.

Aims. By comparing with synthetic observations derived from MHD simulations, we investigate the physical processes underlying the observations, as well as verify the simulations and the interpretation of the observations.

Methods. We consider the temporal evolution of the relevant physical quantities for three cases of magnetic field intensification in a numerical simulation. In order to compare with observations, we calculate Stokes profiles and take into account the spectral and spatial resolution of the spectropolarimeter (SP) on board Hinode. We determine the evolution of the intensity, magnetic flux density and zero-crossing velocity derived from the synthetic Stokes parameters, using the same methods as applied to the Hinode/SP observations to derive magnetic field and velocity information from the spectro-polarimetric data.

Results. The three events considered show a similar evolution: advection of magnetic flux to a granular vertex, development of a strong downflow, evacuation of the magnetic feature, increase of the field strength and the appearance of the bright point. The magnetic features formed have diameters of $0.1-0.2^{\prime \prime}$. The downflow velocities reach maximum values of $5-10 \mathrm{~km} \mathrm{~s}^{-1}$ at $\tau=1$. In the largest feature, the downflow reaches supersonic speed in the lower photosphere. In the same case, a supersonic upflow develops approximately $200 \mathrm{~s}$ after the formation of the flux concentration. We find that synthetic and real observations are qualitatively consistent and, for one of the cases considered, also agree very well quantitatively. The effect of finite resolution (spatial smearing) is most pronounced in the case of small features, for which the synthetic Hinode/SP observations miss the bright point formation and also the high-velocity downflows during the formation of the smaller magnetic features.

Conclusions. The observed events are consistent with the process of field intensification by flux advection, radiative cooling, and evacuation by strong downflow found in MHD simulations. The quantitative agreement of synthetic and real observations indicates the validity of both the simulations and the interpretations of the spectro-polarimetric observations.
\end{abstract}

Key words. Sun: photosphere - Sun: granulation - magnetic fields

\section{Introduction}

Magnetic field is ubiquitously present in the solar photosphere (de Wijn et al. 2008). On granular scales, it undergoes continual deformation and displacement. It is swept by the horizontal flows and concentrated in the intergranular lanes. Flows are able to compress the field so that the magnetic energy density $B^{2} / 8 \pi$ approaches the kinetic energy density $\rho v^{2} / 2$ of the flow (Parker 1963; Weiss 1966). This results in a magnetic field strength of a few hundred Gauss at the solar surface. Further intensification to $\mathrm{kG}$ strength is driven by the mechanism referred to as: $s u$ peradiabatic effect (Parker 1978), convective collapse (Webb \& Roberts 1978; Spruit \& Zweibel 1979) or convective intensification (Grossmann-Doerth et al. 1998).

The first two concepts are a theoretical idealization of the process. The superadiabatic effect contains the basic idea. Parker (1978) pointed out that a thermally isolated dowflowing gas within the flux tube in a superadiabatically stratified environment will be accelerated, which would lead to evacuation of the flux tube. Because of the resulting pressure deficit, the gas inside the flux tube will then be pressed together (together with the frozen-in magnetic field) by the surrounding gas, causing the magnetic pressure to increase until a balance of total pressure (magnetic + gas) is reached.

The convective collapse extends the concept to the convective instability. It starts with a flux tube in thermal and mechanical equilibrium with the surrounding hydrostatically superadiabaticly stratified plasma. Since external stratification is convectively unstable, any vertical motion within the flux tube can be amplified. Downward flow will grow in amplitude and drain the material from the flux tube. The process continues until a new equilibrium with a strong field is reached. Different aspects of the concept have been the subject of extensive research (see Schüssler 1990; Steiner 1999, for reviews).

The term convective intensification is used for magnetic field intensification in realistic MHD simulations, where the process occurs in its full complexity. It is driven by the thermal effect in the surface layer of the magnetic concentration. There, due to the presence of the magnetic field, heat transport by convection is reduced. The material inside the concentration radiates more that it receives. This leads to cooling of material which thus starts to sink and partial evacuation of the concentration occurs. Contraction of the magnetic concentration by the surroundings (a result of the pressure imbalance) leads to an increase in 

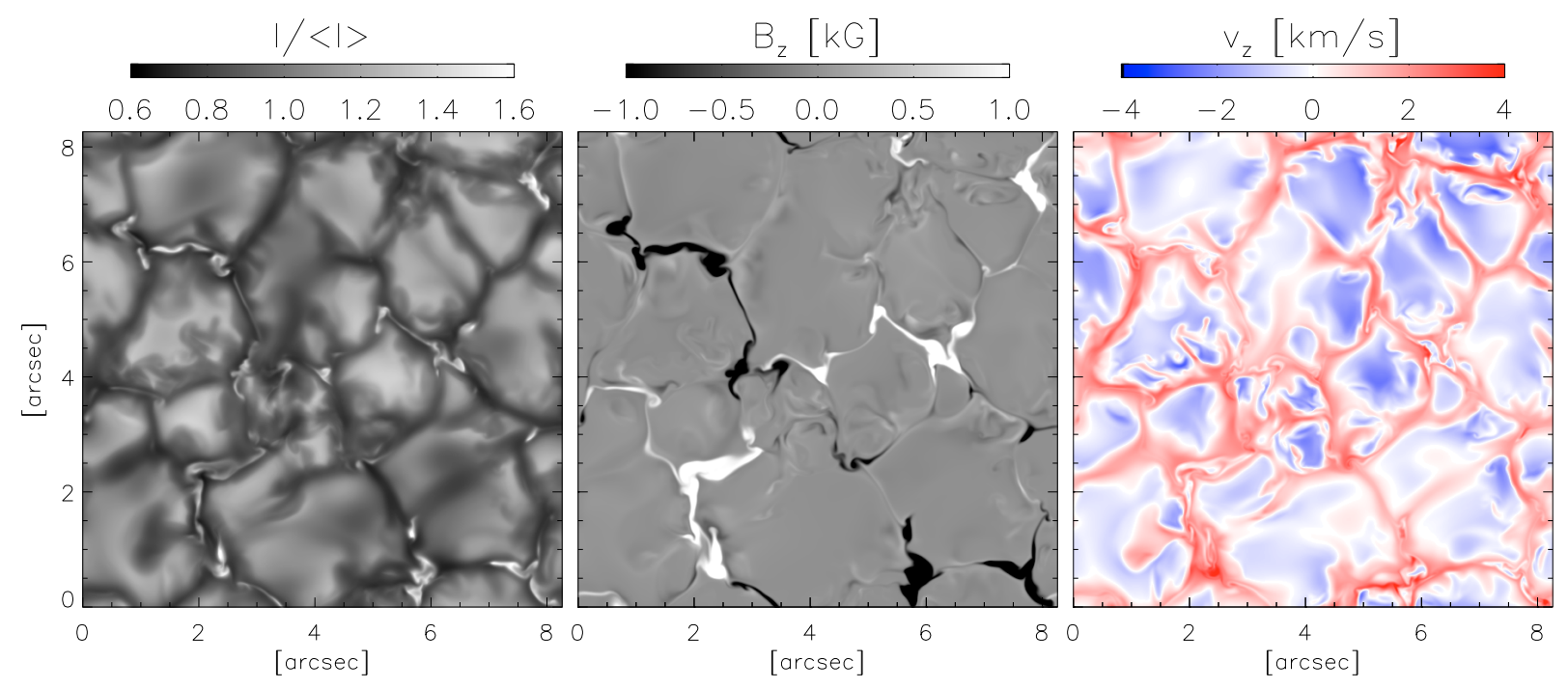

Fig. 1. Maps of the whole simulation domain at $t=140 \mathrm{~s}$. Normalized continuum intensity at $630 \mathrm{~nm}$ (left), the vertical component of the magnetic field (middle) and velocity (right) at a geometrical height roughly corresponding to the level of $\left\langle\tau_{500}\right\rangle=1(\approx 930 \mathrm{~km}$ above the bottom of the computational box).

magnetic field strength. Thus, the simulations (Nordlund 1983; Grossmann-Doerth et al. 1998; Gadun et al. 2001; Vögler et al. 2005; Cheung et al. 2008) bear out the basic properties described by idealized concepts, such as: the downflow, the evacuation of the magnetic structure, the field increase and, in some cases, establishment of a new equilibrium. The 3D MHD simulations show that the strong magnetic concentrations form as the horizontal flows in the intergranular lanes advect the weak, nearly vertical field and concentrate it at the vertices of granular and mesogranular downflow lanes (Stein \& Nordlund 1998, 2006). Larger magnetic structures form at sites where a granule submerges and the surrounding field is pushed into the resulting dark region. Whether the concentration formed appears dark or bright in the continuum intensity depends on whether the vertical cooling is compensated or not by the lateral heating due to horizontal energy exchange (Bercik et al. 2003; Vögler et al. 2005). This formation scenario is consistent with the observations described by Muller (1983) and Muller \& Roudier (1992). Their observations show that network bright points form in intergranular spaces, at the junction of converging granules as the magnetic field becomes compressed by the converging granular flow.

The 2D simulations by Grossmann-Doerth et al. (1998) revealed that magnetic flux concentrations formed by convective intensification can evolve in different ways. They present two possible outcomes. Depending on the initial magnetic flux, a magnetic concentration can reach a stable state after the process, or can be dispersed due to an upflow that develops as high speed downflowing material rebounds from the dense bottom of the tube. Similar results were presented by Takeuchi (1999) and Sheminova \& Gadun (2000).

Observational evidence was found for both cases. Bello González et al. (2008) reported on the formation of a magnetic feature at the junction of intergranular lanes, without any significant upflow observed. Bellot Rubio et al. (2001), on the other hand, detected a strongly blueshifted Stokes $V$ profile originating in a upward propagating shock, 13 minutes after the amplification of the magnetic field. Socas-Navarro \& Manso Sainz (2005) found that supersonic upflows are actually quite common.
Events that are interpreted as convective collapse were detected also with the spectropolarimeter (SP) (Lites et al. 2001) of the Solar Optical telescope (Tsuneta et al. 2008) on board Hinode (Kosugi et al. 2007). Shimizu et al. (2008) and Nagata et al. (2008) show cases of high speed downflows followed by magnetic field intensification and bright point appearance. The event described by Nagata et al. (2008) shows stronger field strength and upflow in the final phase of evolution.

In this paper we give three examples of magnetic field intensification from MURaM simulations and perform a detailed comparison with the results of Nagata et al. (2008) and Shimizu et al. (2008).

\section{Simulation data and spectral synthesis}

We use 3D radiative MHD simulations of a thin layer containing the solar surface carried out with the MURaM code (Vögler 2003; Vögler et al. 2005) in a $6 \times 6 \times 1.68 \mathrm{Mm}$ domain with nongrey radiative transfer included. Vertical and horizontal spatial resolution is $10 \mathrm{~km}$ and $14 \mathrm{~km}$ respectively. The bottom and top boundaries are open, permitting free in and outflow of matter. The initial magnetic field of $\langle|B|\rangle=200 \mathrm{G}$ is introduced in a checkerboard-like $2 \times 2$ pattern, with opposite polarity in adjacent parts. As the field is redistributed by convective motion, opposite polarities are pushed together and dissipated, so that the unsigned magnetic flux decreases with time. In this way, the run simulates the decay of the magnetic field in a mixed polarity region. Local dynamo action (Vögler \& Schüssler 2007) does not occur since the magnetic Reynolds number is below the threshold for dynamo action. We do not expect that the intensification process would be significantly different at higher Reynolds numbers (smaller grid cells). The simulated magnetic flux concentrations considered here are well resolved. The main effect of an increased resolution would be a decrease in the width of the boundary layers between the flux concentration and the surrounding downflows.

We examined a 30 min sequence of simulation snapshots with a cadence of approximately $90 \mathrm{~s}$. The snapshots shown in this paper have $\langle|B|\rangle \approx 150 \mathrm{G}$ at $\tau=1$. In Fig. 1, we show the continuum image and maps of the vertical components of the magnetic field and velocity for a snapshot at time $t=140 \mathrm{~s}$ 

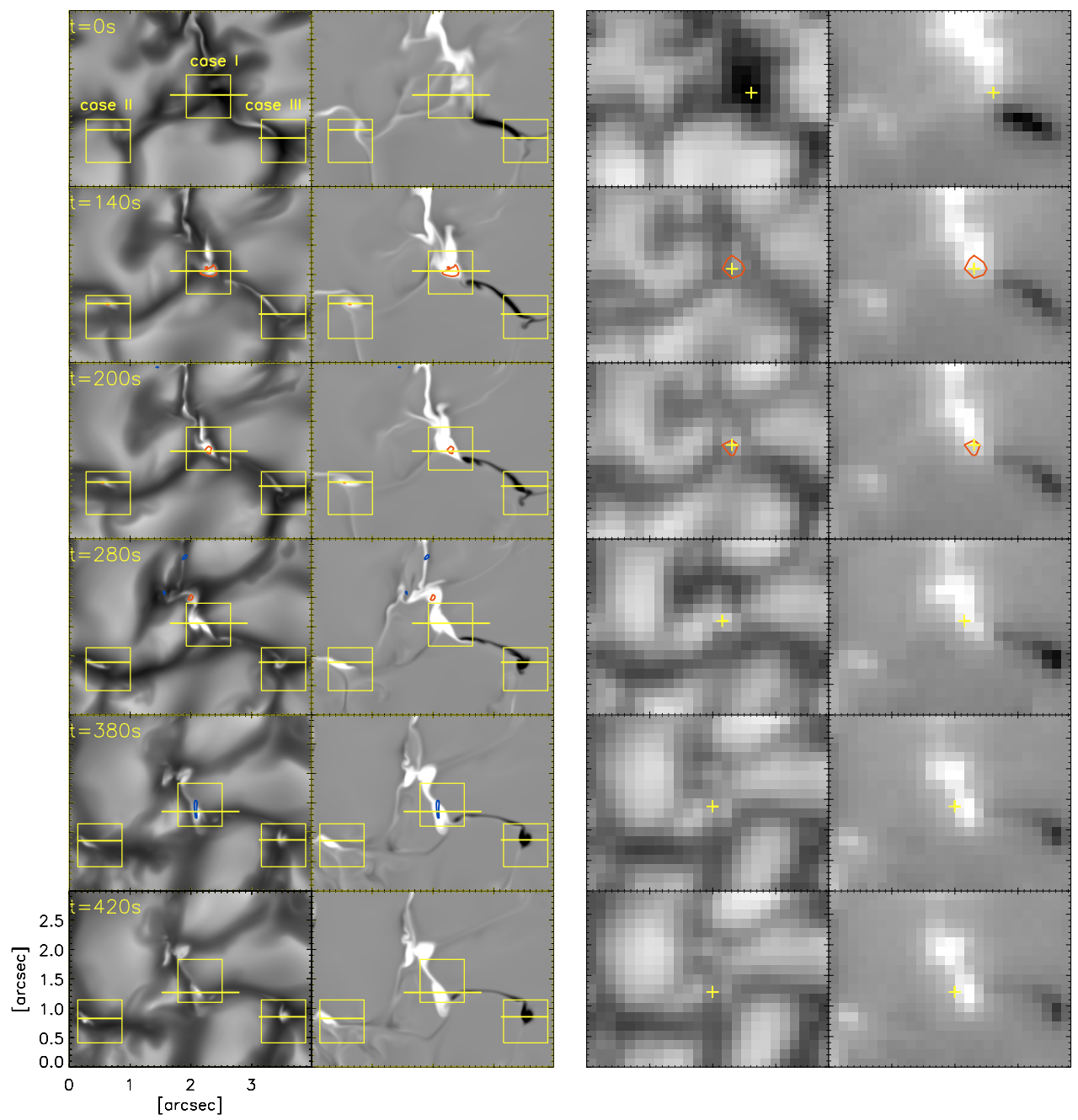

Fig. 2. Evolution of the continuum intensity at $630 \mathrm{~nm}$ and the magnetic field in a 4 " $\times 3^{\prime \prime}$ sized region. Left-hand side (double column): original spatial resolution; red and blue contours outline the downflow of $6 \mathrm{~km} \mathrm{~s}^{-1}$ at $80 \mathrm{~km}$ and upflow of $4 \mathrm{~km} \mathrm{~s}^{-1}$ at $400 \mathrm{~km}$ above $\tau=1$, respectively; horizontal lines mark the positions of vertical cuts shown in Figs. 4 and 6; boxes corresponding to cases I, II and III at coordinates [2", 1.5"1'], $\left[0.5^{\prime \prime}, 1^{\prime \prime}\right]$ and $\left[3.5^{\prime \prime}, 1^{\prime \prime}\right]$, respectively, enlarged in Fig. 3. Right-hand side: synthetic Hinode/SP observations; the left column shows continuum intensity while the right column shows the apparent field strength (see the text); the red contour marks the region with $0.01 \mathrm{pm}$ of signal excess (see text); yellow crosses mark the positions of pixels that we studied in detail in Fig. 9. The grey scale covers the range of $0.6-1.6$ and $0.8-1.2$ for normalized intensity at original and reduced resolution, respectively, $\pm 1000 \mathrm{G}$ for the vertical component of magnetic field (original resolution) and $\pm 200 \mathrm{Mx} / \mathrm{cm}^{2}$ for the apparent longitudinal magnetic flux density (Hinode resolution).

$(t=0 \mathrm{~s}$ corresponds to the first snapshot considered in this paper).

In order to synthesize the Stokes profiles, the physical parameters from the simulation are used as an input for the 1D LTE radiative transfer code, SPINOR (Frutiger et al. 2000). A spectral range that contains Fe I lines 630.15 and $630.25 \mathrm{~nm}$ is sampled in steps of $7.5 \mathrm{~m} \AA$. The Fe abundance used for the synthesis has been taken from Thevenin (1989) and the values of the oscillator strengths from the VALD database (Piskunov et al. 1995). Before comparing with Hinode/SP (Lites et al. 2001) observations, the synthetic line profiles have been treated to bring them to the same resolution as Hinode data. Firstly, a realistic point spread function (PSF, Danilovic et al. 2008) was applied to the synthesized Stokes profiles. The PSF takes into account the basic optical properties of the Hinode SOT/SP system and a slight defocus which brings the continuum contrast of the simulation to the observed value of $7.5 \%$. Secondly, to take into account the spectral resolution of the spectropolarimeter, the profiles are convolved with a Gaussian function of $25 \mathrm{~m} \AA F W H M$ and resampled to a wavelength spacing of $21.5 \mathrm{~m} \AA$. Thirdly, noise of $10^{-3} I_{\mathrm{c}}$ is added. Velocities are determined from the shift between the Stokes $V$ zero-crossing wavelength and the line core position of the Stokes I profile averaged over pixels with polarization signal amplitudes less than $10^{-3} I_{\mathrm{c}}$. We correct these velocities for convective blueshift by subtracting $150 \mathrm{~m} \mathrm{~s}^{-1}$. Finally, the procedure by Lites et al. (2008) is used to calculate the longitudinal apparent magnetic flux density (shown in Fig. 2).

\section{Results}

Figure 2 shows the evolution of the continuum intensity and the magnetic field in a $2.9 \times 2.2 \mathrm{Mm}$ subdomain of the simulation, over approximately $7 \mathrm{~min}$. Maps at the original resolution of the simulation (left-hand side) and at the spatial resolution of Hinode (right-hand side) are shown. For the original resolution, we show the vertical component of the magnetic field from the simulations (right column on the left-hand side). The 


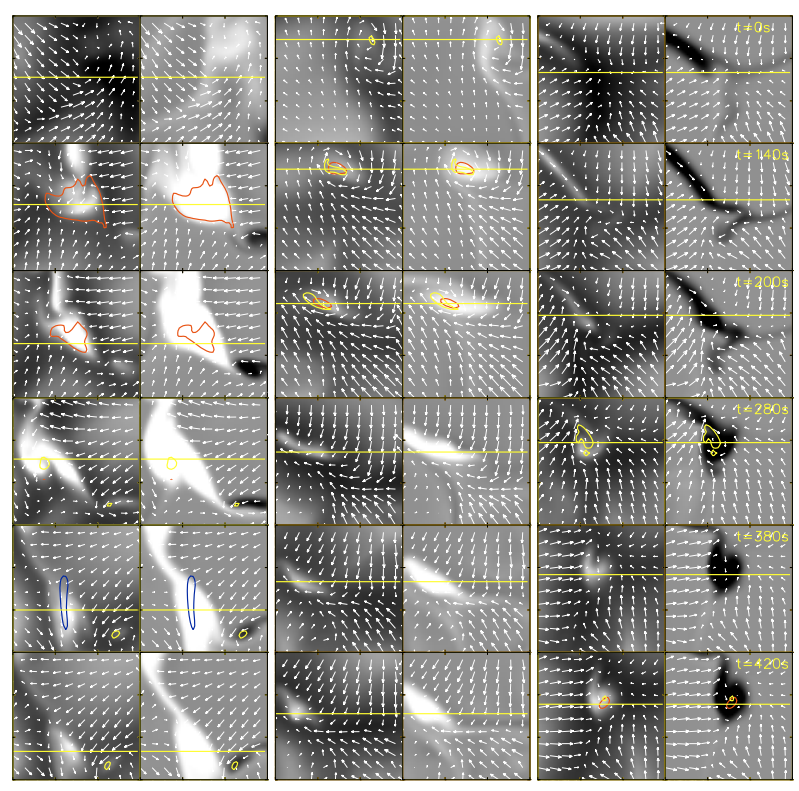

Fig. 3. Enlargement of the regions, having a size of $0.75^{\prime \prime} \times 0.75^{\prime \prime}$, outlined by yellow squares in Fig. 2. From left to right: cases I, II and III. Continuum images (left column for each case) and maps of the vertical components of the magnetic field (right) with overplotted horizontal velocities at approximately $80 \mathrm{~km}$ above $\tau=1$ (white arrows). Red contours outline locations of dowflows of $5 \mathrm{~km} \mathrm{~s}^{-1}$ and yellow contours the vertical component of vorticity exceeding $\pm 0.2 \mathrm{~s}^{-1}$ (cases I and II) or $\pm 0.1 \mathrm{~s}^{-1}$ (case III).

longitudinal apparent flux density (Lites et al. 2008) retrieved from synthetic Stokes profiles is shown at the Hinode resolution (right column on the right-hand side). During the period shown, three bright points appear near the coordinates $\left[2^{\prime \prime}, 1.5^{\prime \prime}\right]$, $\left[0.5^{\prime \prime}, 1^{\prime \prime}\right]$ and $\left[3.5^{\prime \prime}, 1^{\prime \prime}\right]$, in the regions outlined by yellow squares. We refer to them here as cases I, II and III, respectively. They are identified in the top left frame.

\subsection{Horizontal flows}

In all three cases, the magnetic field is advected by the flow to the junction of multiple granules, where it is confined and concentrated. The evolution of magnetic concentrations is examined more closely in Fig. 3, where the regions outlined by yellow squares in Fig. 2 are enlarged. Horizontal velocities at approximately $80 \mathrm{~km}$ above $\langle\tau\rangle=1$ are represented by arrows in continuum maps and magnetograms. Regions with a strong vertical component of vorticity are also outlined.

Vortex flows around strong downflows are quite common in simulations of granulation (Nordlund 1986). They are formed at the vertices between multiple granules, where flows converge and angular velocities with respect to the center of downflow increase due to angular momentum conservation. The lifetime of vortex flows depends on the dynamical behavior of the neighboring granules. In a 30 min run that we examined, they lasted from less then $90 \mathrm{~s}$ to approximately $10 \mathrm{~min}$. The ones shown in Fig. 3 are short-lived. In case II, the swirling motion persists for at least $150 \mathrm{~s}$, until the shape of the neighboring granules is changed. The flux concentration formed is then squeezed between two granules and stretched into a flux-sheet-like feature. Later, as the granules evolve, the magnetic feature is advected to the left and trapped again in the junction between newly formed granules. In case III, a strong preexisting flux sheet is carried by the flow toward the junction of the granules. There, the field is caught in a vortex flow that lasts for at least $100 \mathrm{~s}$. The flow becomes disturbed and then starts to swirl in the opposite direction. The vortex axes are inclined, in both cases II and III, with respect to the vertical direction. Also in both cases, there is a spatial and temporal coincidence between the existence of vortex flows and the formation of high speed downflows near the surface layer.

The vortices shown in Fig. 3 are less than $0.25^{\prime \prime}$ in diameter, much smaller then the ones found in observations (Bonet et al. 2008). The size of the observed vortex flows is of the order of $1^{\prime \prime}$ and the average lifetime is around $5 \mathrm{~min}$. They seem to outline supergranulation and mesogranulation cells. As the authors of the observational study suggest, most vortices could have been missed because of the limited spatial resolution (Swedish Solar Telescope) and the method used (tracking the motion of bright points in $\mathrm{G}$ band). A short-lived vortex flow preceding formation of a network bright point has been observed by Roudier et al. (1997).

No vortex is detected during the formation of flux concentration in the case I. In this case, a strong downflow and later upflow develops inside the flux concentration, whose shape and contrast in the intensity map is greatly affected by the convective motion. Spatial and temporal fluctuations across the magnetic feature make it difficult to define its center. The bright points, visible in continuum maps, form where fragments of flux concentrations become narrow so that lateral heating becomes important, as well as in the regions with higher field strength, i.e. higher evacuation.

During their further evolution after the intensification phase $(t>420 \mathrm{~s})$, the flux concentrations in cases I, II and III have different fates. In case II, it meets a magnetic feature of opposite polarity and vanishes in $\sim 3 \mathrm{~min}$. The feature formed in case I is gradually stretched by the flow and fragmented after approximately 9 min. A few minutes after that, the larger fragment encounters the opposite-polarity flux concentration from case III. The cancellation continues for $\sim 12$ min until both features disappear. The smaller fragment of the feature from case I is caught in a granular vortex flow approximately 5 min later which leads to further intensification.

\subsection{Field intensification}

The evolution of the magnetic structures formed during the studied time interval can be followed in more detail by taking vertical slices through the regions marked by horizontal lines in Fig. 3. We will examine three cases.

\subsubsection{Case I}

Vertical cuts through the regions corresponding to this case are given in Fig. 4. The density excess (relative to the mean density at each geometrical height), the vertical components of magnetic field and velocity, and the radiative heating rate are shown. Cuts at the instant $t=0$ show the fluctuation in density outlining the granulation structure, with less dense, hotter granules and a cooler intergranular lane, where magnetic field of a few hundred $\mathrm{G}$ is accumulated and a downflow of a few $\mathrm{km} / \mathrm{s}$ is present. The fluctuation in radiative heating rate is highest in the thin layer near an optical depth unity, where upflowing granular material cools most strongly. The regions along the intergranular lane seems to undergo cooling in the layers above $\tau=1$. In the next instant, $140 \mathrm{~s}$ later, a strong magnetic concentration has already formed. Variations in magnetic field strength at a given geometrical height are accompanied by variations in the gas 


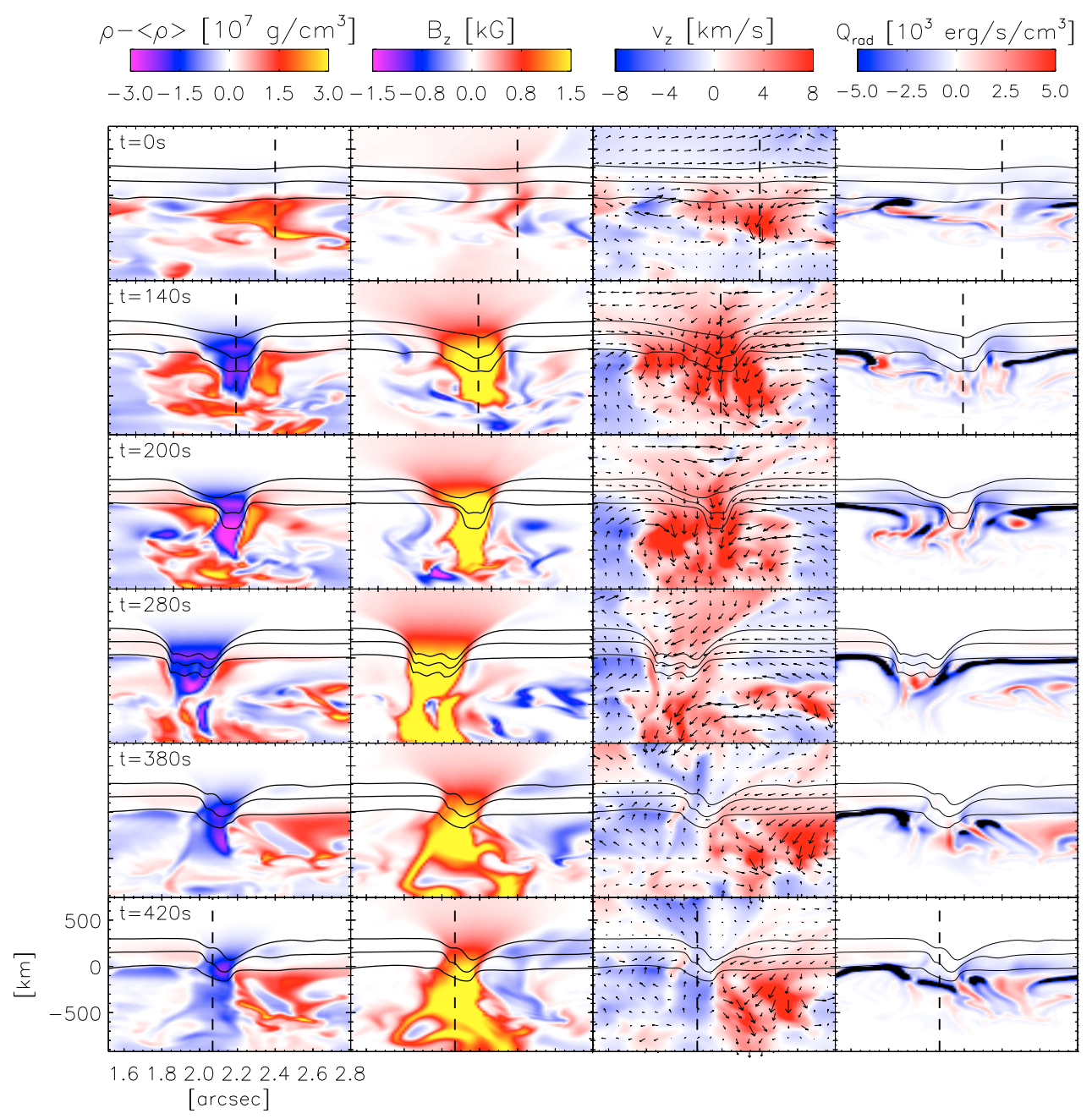

Fig. 4. Time sequence of vertical cuts through the magnetic concentration corresponding to case (positions of the cuts are marked by yellow horizontal lines in right-hand images in Fig. 2). The plotted cuts correspond to the same instants as sampled in Fig. 2. From left to right: density excess with respect to the the mean density at every height, vertical component of magnetic field, vertical component of velocity (overplotted arrows show the components of the velocity field in the $x-z$ plane), and radiative heating rate. Horizontal solid lines follow the levels of log $\tau=0,-1$ and -2 . Vertical dashed lines mark the position of the height profiles given in Fig. 5.

pressure. Regions with increased field strength have lower density, which in turn shifts the level of optical depth unity downwards relative to neighboring regions with weaker field. The depression of the visible surface inside the magnetic structure gives rise to radiative heating through the sidewalls from the hot neighboring material. The downflow velocity is significantly increased from $t=0$ to $t=140 \mathrm{~s}$, becoming supersonic in the layer between $100 \mathrm{~km}$ above and $350 \mathrm{~km}$ below the surface. By $t=280 \mathrm{~s}$ it is greatly diminished again inside the flux concentration. The diameter and the shape of the flux concentration continuously evolves and does not reach a stationary state. At $t=280 \mathrm{~s}$ strong radiative heating is present inside the concentration. This is also the moment when the continuum intensity is the highest, as shown later (Fig. 9).

In the last two sets of snapshots shown in Fig. 4, an upflow is visible inside the flux concentration, extending almost from the bottom of the simulation domain. It reaches supersonic velocities in the upper layers of the photosphere. Its direction is inclined with respect to the vertical. The upflowing material inside the concentration exhibits strong cooling at the surface. As the material refills the region inside the flux concentration, the level of optical depth unity shifts upwards and the magnetic field strength decreases. However, the upflow does not lead to a complete dispersal of the field, as described in the last paragraph of Sect. 3.1.

The evolution of this flux concentration is further illustrated by the vertical profiles given in Fig. 5. Three phases before and after the field intensification are shown. The horizontal positions of the profiles are marked by vertical dashed lines in Fig. 4. The profiles of magnetic field strength, the vertical component of velocity, the density and the temperature for each phase are shown, together with the corresponding level of optical depth unity, marked by thick vertical lines. The level $h=0$ corresponds to the level of the mean optical depth unity $\left\langle\tau_{500}\right\rangle=1$ for the whole snapshot. Thin vertical lines in the upper plots mark the position of the average height of formation of the Fe I $630.25 \mathrm{~nm}$ line, which is defined by calculating the centroid of the contribution function $(\mathrm{CF})$ for line intensity depression (Solanki \& Bruls 1994), at the wavelength of the line core. The definition gives only a rough estimate of the heights sampled, since the lines are formed over a large portion of the photosphere and the CFs are asymmetric in wavelength due to the presence of high velocity gradients.

The figure shows that, in the interval from $t=0 \mathrm{~s}$ to $t=140 \mathrm{~s}$, the downflow extends to deeper layers and increases in amplitude, reaching $10 \mathrm{~km} \mathrm{~s}^{-1}$ at $200 \mathrm{~km}$ below the surface. The 

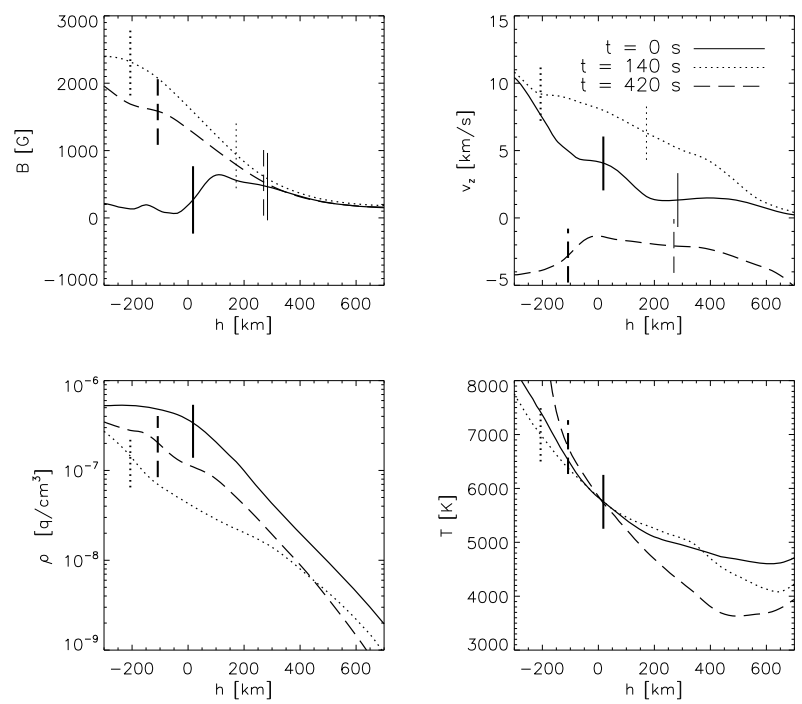

Fig. 5. Height profiles of various quantities in the flux concentration corresponding to the case I (magnetic field, vertical component of velocity, density and temperature) at the positions marked by dashed lines in Fig. 4 at different times. Thick vertical lines mark the positions of the optical depth $\tau_{500}=1$ for each atmosphere. Thin vertical lines in the top two panels mark the positions of the height of formation of Fe I $630.25 \mathrm{~nm}$ line (see text).

magnetic field strength increases from a few hundred to more than $2000 \mathrm{G}$ at the level of $\tau_{500}=1$. The significant reduction in density results in a shift of $200 \mathrm{~km}$ in the optical depth unity level. As a result of radiative heating, the temperature gradient is flatter and the temperature is around $400 \mathrm{~K}$ higher at $h=0$. The temperature difference at equal optical depth is even greater, exceeding $1000 \mathrm{~K}$ at $\tau_{500}=1$. The estimated height of formation of Fe I 6302 shifts from roughly $300 \mathrm{~km}$ to $150 \mathrm{~km}$ above the surface when magnetic field concentration forms, which is in agreement with Khomenko \& Collados (2007). This effect contributes to the increase of observed magnetic field strength in the first two phases.

In the third phase, the upflow leads to a density enhancement and a weakening of the magnetic field by a few hundred Gauss in the lower photosphere. The level of $\tau_{500}=1$ shifts upward by $100 \mathrm{~km}$. No discontinuity is visible in the vertical component of velocity, in contrast to the event studied by Grossmann-Doerth et al. (1998).

\subsubsection{Cases II and III}

Vertical cuts through the regions corresponding to these cases are shown in Fig. 6. The positions of the cuts are marked by horizontal lines in Fig. 3. The parameters shown are the same as in Fig. 4.

At $t=0$, weak magnetic structures in the intergranular lanes are already present in both cases. Strong downflows along the magnetic field lines persist over the entire time interval shown. They reach supersonic velocities in some instances, but only in the layers below $\tau=1$. In case II, the downflow is strongest at $t=140 \mathrm{~s}$, just before the vortex flow becomes deformed owing to evolution of the neighboring granule. Then it becomes somewhat reduced at $t=280 \mathrm{~s}$, as the structure is squeezed between the granules. Later, at $t=380 \mathrm{~s}$, a strong downflow is present again in the newly formed granular junction, to which the flux concentration is carried and where it is confined again. The rightmost columns show strong cooling of the layers above the level of $\tau_{500}=1$ in both cases. At $t=420 \mathrm{~s}$, the interior of the flux concentrations reaches radiative equilibrium. At that moment, the flux concentrations in both cases are almost vertical, with diameters of approximately $0.1^{\prime \prime}$ at $\tau_{500}=1$.

Figures 7 and 8 show height profiles along the positions marked by the dashed lines in Fig. 6. Since the flux concentrations are inclined, the choice of curved lines along the axes of magnetic structures would be more logical here. However, we are interested in what would be observed at the disc center and the observables (i.e. the Stokes profiles) depend on the vertical profiles of the physical parameters which are shown here. In the case II, we choose to show profiles at instants $t=0,280$ and $420 \mathrm{~s}$ (solid, dotted, dashed respectively). For the case III, profiles at the last three instants in Fig. 6 are shown. Both cases exhibit a similar behavior: there is a significant evacuation of the magnetic concentration due to the strong downflow and an increase of the magnetic field strength from a few hundred $G$ to $\mathrm{kG}$ values in the process. There is a persistent dowflow that reaches $5 \mathrm{~km} \mathrm{~s}^{-1}$ (in the vertical direction) at $\tau_{500}=1$ and a decrease in density with a corresponding shift in the height of $\tau_{500}=1$. In both cases, the shift in $\tau_{500}=1$ reaches $150 \mathrm{~km}$ and the magnetic field strength increases to $2000 \mathrm{G}$ at that depth. The height of formation of the Fe I $630.25 \mathrm{~nm}$ line shifts to deeper layers as the magnetic field strength increases. In the last instant, the Fe I $630.25 \mathrm{~nm}$ line probes layers with $\mathrm{kG}$ fields and a vertical velocity of $5 \mathrm{~km} \mathrm{~s}^{-1}$ in both cases.

\subsection{Comparison with synthetic Hinode observations}

The intensity contrast is considerably reduced at Hinode spatial resolution, as can be seen in Fig. 2 (note that the color scales are adjusted). The maps of longitudinal apparent magnetic flux density are shown for comparison with the magnetograms at the original resolution. Fine structure in both maps, continuum and magnetograms, is lost due to smearing. The bright points that correspond to cases II and III are barely discernible.

\subsubsection{Case I}

In order to compare case I with the results of Shimizu et al. (2008), we calculate the parameter they refer to as signal excess (SE). It is defined as the Stokes $V$ profile integrated over the spectral range of 250-400 $\mathrm{m} \AA$ redward from the nominal line center. This wavelength range corresponds to dowflows of $7-14 \mathrm{~km} \mathrm{~s}^{-1}$, so that high values of SE indicate the presence of hight-speed downflows. The red contour in the right-hand side of Fig. 2 outlines a region with an SE of $0.01 \mathrm{pm}$, which is a factor of 10 higher than in the surroundings. It fits the location and time of the strong downflows visible at the original resolution (left hand-side of Fig. 2). Both synthesized (here) and observed (Shimizu et al. 2008) SE occur simultaneously with the appearance of the bright point and the intensification of the magnetic field. However, the event described by Shimizu et al. (2008) (Fig. 8) lasts at least $6 \mathrm{~min}$, while in our simulations it is present for less than $4 \mathrm{~min}$.

Figure 9 compares the observable parameters with the corresponding values at original resolution. The locations chosen for the plots are marked by yellow crosses in Fig. 2. The pixels are selected such that regions of downflow and upflow are covered, as well as the evolution of the bright point. The plots show the temporal change of normalized intensity, longitudinal apparent flux density (Lites et al. 2008), and zero-crossing velocity retrieved from Stokes $V$ profiles of the Fe I $630.25 \mathrm{~nm}$ line, so that 


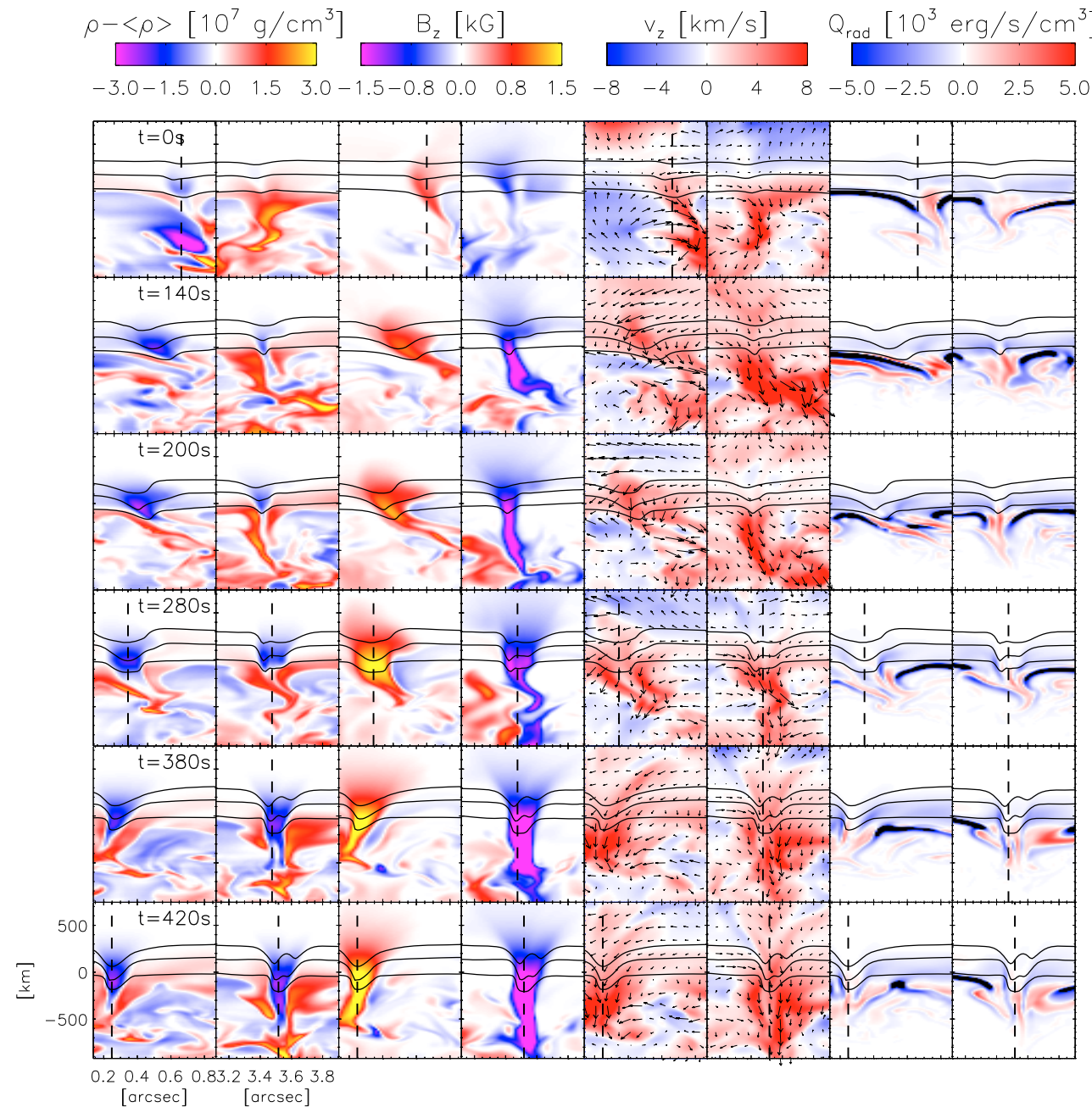

Fig. 6. The same as Fig. 4 but for cases II and III. Each frame is subdivided into a left part displaying case II and a right part showing case III. Vertical lines mark the positions of the height profiles given in Figs. 7 and 8 .
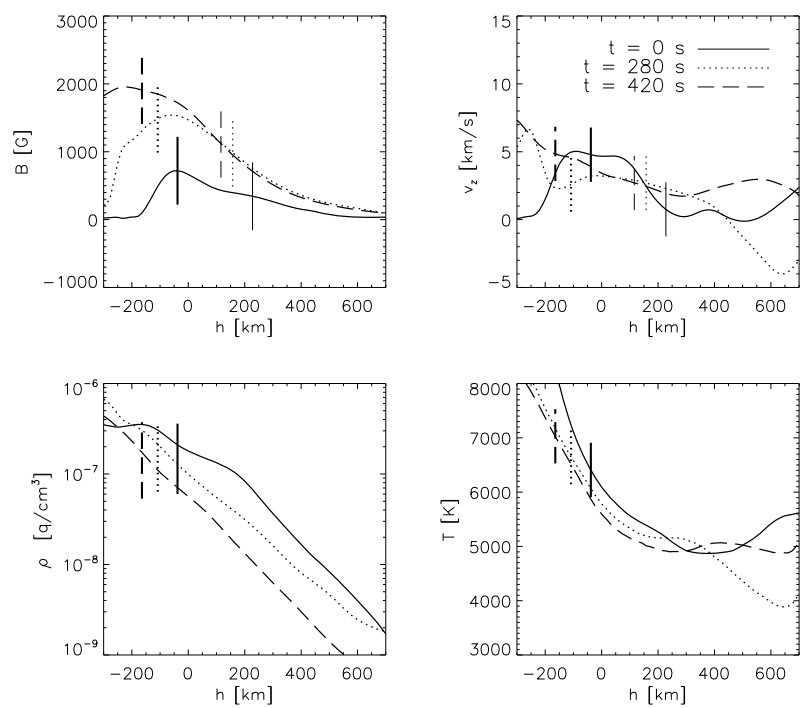

Fig. 7. The same as Fig. 5, but for case II.

a direct comparison with the results of Nagata et al. (2008) can be made. Overplotted (stars/solid lines) are the normalized intensity at the original resolution, the vertical component of the magnetic field and velocity at $\log \tau_{500}=-2$.
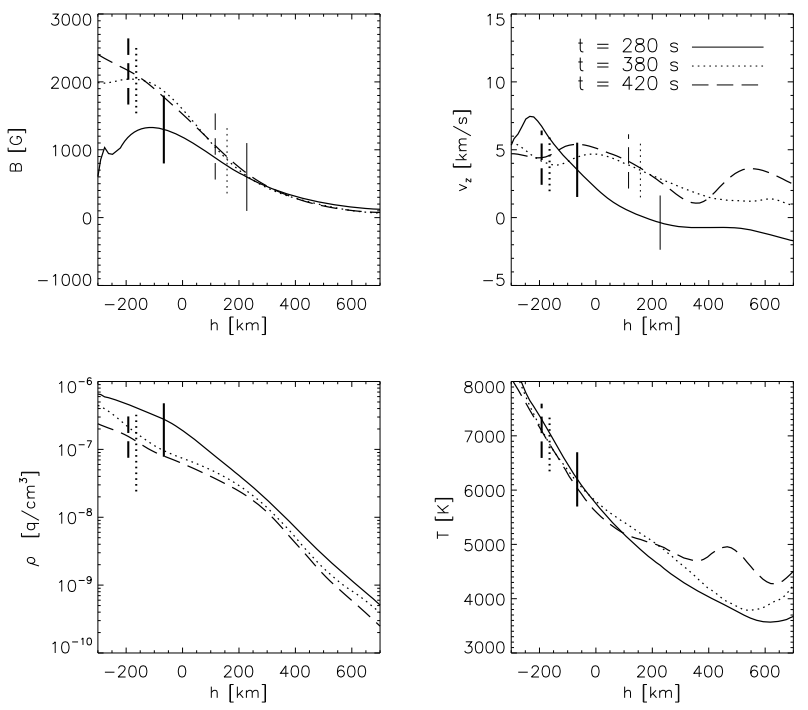

Fig. 8. The same as Fig. 5, but for case III.

The comparison shows that the correlation between the parameters at original and reduced resolution is not so good. This is a consequence of different effects. Firstly, since the formation region of the Fe I $630 \mathrm{~nm}$ lines extends over a large part of the 

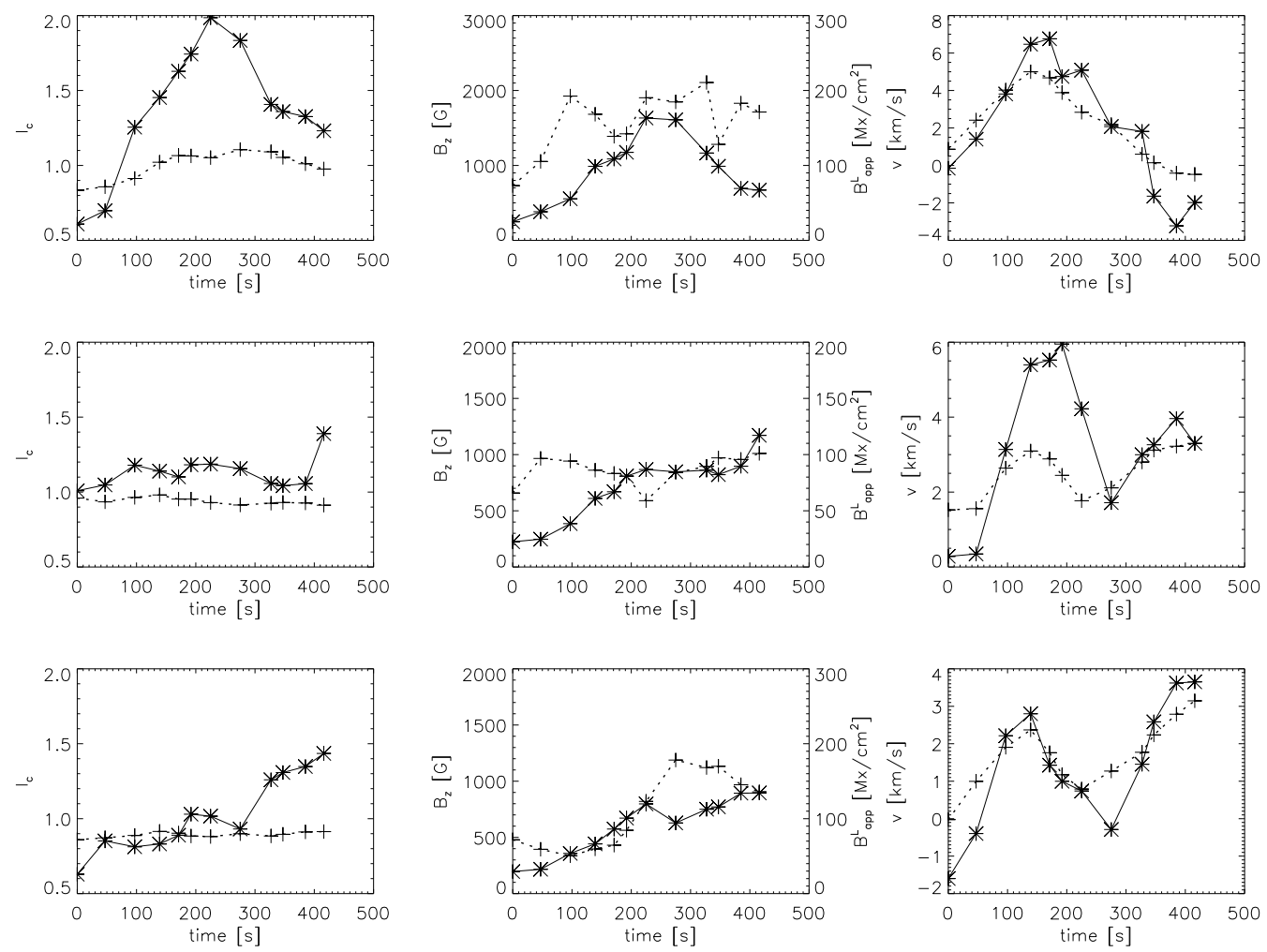

Fig. 9. Temporal change of normalized continuum intensity (left), the vertical component of magnetic field (middle) and the vertical component of velocity (right), for cases I (top row), II (middle row) and III (bottom row) at original (stars/solid line) and Hinode resolution (crosses/dashed line). The vertical component of the magnetic field and velocity at $\log \tau_{500}=-2$ (for original resolution) are each plotted together with the longitudinal apparent magnetic flux density (note the separate scale employed for $B_{\text {app }}^{\mathrm{L}}$ in the middle column) and the velocity retrieved from Stokes $V$ profiles (Hinode resolution), respectively.

atmosphere, there is smearing along the line of sight and the resulting profiles carry information from different layers with different values of the physical parameters. Secondly, the smearing due to spatial resolution affects every pixel differently, depending on its neighboring pixels. The profiles could be more asymmetric and broader due to averaging over inhomogeneous regions and the amplitude of Stokes $V$ can be affected. Figure 10 shows examples of Stokes $I$ and $V$ profiles at original and reduced resolution at $t=0,140$ and $420 \mathrm{~s}$. The profiles show stronger asymmetries and higher Doppler shifts at the original resolution. The amplitudes of Stokes $V$ profiles at Hinode resolution are reduced by a factor of 10 . They are of the same order of magnitude as the observed ones (Fig. 2 of Nagata et al. (2008)). Both synthesized and observed Stokes I profiles show an extended red wing in the phase with strong downflow.

Figure 9 shows that intensity contrast is significantly reduced by spatial smearing. Thus at $t=230 \mathrm{~s}$ the contrast of the bright point drops from $100 \%$ at the original resolution to below $30 \%$ at the reduced resolution. The velocity determined from Stokes $V$ profiles is at most times somewhat lower than the velocities at $\log \tau_{500}=-2$. However, the general trends of the parameters are preserved. For both simulation and observation (Nagata et al. 2008), the intensity reaches its maximum approximately $100 \mathrm{~s}$ later than velocity. This is consistent with the idea that the enhanced brightness results from the evacuation due to the downflow. When the velocity changes sign, the brightness starts to fade. The maximum brightness of the simulated and observed bright points are similar after degrading the simulated intensity to Hinode resolution, while the velocities derived from the simulations are consistent, but somewhat smaller. They reach approximately $5 \mathrm{~km} \mathrm{~s}^{-1}$ (downflow) and $0.5 \mathrm{~km} \mathrm{~s}^{-1}$ (upflow), while
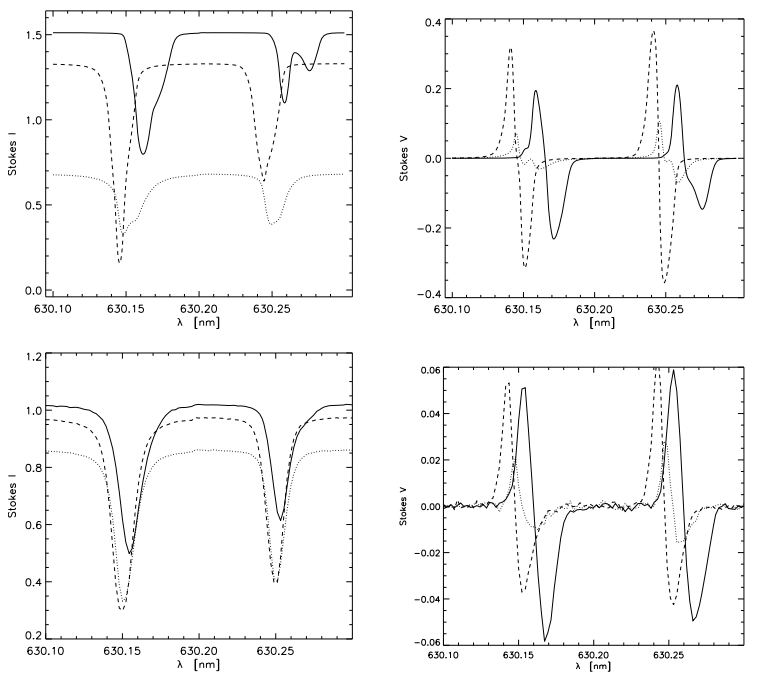

Fig. 10. Synthetic Stokes I (left) and $V$ (right) profiles at original (upper row) and Hinode resolution (lower row) for the times $t=0,140$ and $420 \mathrm{~s}$ marked by dotted, solid and dashed lines, respectively. The intensity is normalized to the mean continuum intensity in the full snapshot domain.

values up to $6 \mathrm{~km} \mathrm{~s}^{-1}$ and $2 \mathrm{~km} \mathrm{~s}^{-1}$, respectively, are observed. The magnetic field strength obtained by Nagata et al. (2008) by inversions increases from a few hundred to $2000 \mathrm{G}$ in approximately $200 \mathrm{~s}$, which is in agreement with simulations. 


\subsubsection{Cases II and III}

The middle and bottom rows in Fig. 9 show the temporal evolution for cases II and III, respectively. We find a simultaneous increase in all parameters (continuum intensity, apparent flux density and zero-crossing velocity). In both cases, the downflow is suppressed at $t=280 \mathrm{~s}$, possibly because of the evolution of the surrounding granules. The velocity at $\log \tau_{500}=-2$ reaches 6 and $4 \mathrm{~km} \mathrm{~s}^{-1}$ for case II and III, respectively. As the magnetic field strength increases, the intensity follows and, in both cases, the intensity contrast reaches $50 \%$ with respect to the mean at original resolution.

The influence of spatial smearing is on the whole similar, although a few differences relative to case I are noteworthy. Firstly, due to the small size of the features, the correlation of the magnetic and brightness signals is largely destroyed. Although the magnetic signals are comparable to the values in case I, the brightness is lower, so that the features are inconspicuous in continuum images. The increase in magnetic flux density is followed by only a small fluctuation in intensity. Secondly, although the magnetic field strengths and diameters of the features are comparable for cases II and III at the original resolution, the case II shows lower magnetic flux density after convolution. Thirdly, the signature of the strong dowflow in case II is lost due to spatial smearing.

\section{Summary}

Our case study of three examples of magnetic field intensification has shown that in all three cases, the field is advected to the junction of several granules. There, it is confined by converging granular flows, which, in two cases, form a vortex. Due to the presence of the magnetic field, the thermal effect (radiative cooling) induces evacuation of the flux concentration. The evacuation leads to a downward shift of the optical depth scale within the flux concentrations. The shift is smaller for the smaller features due to the lateral radiative heating, which inhibits further evacuation (Venkatakrishnan 1986). As a result, the magnetic field at $\tau=1$, in the smaller features, is weaker than in the case of the feature with more flux. This is in accordance with recent numerical (Cheung et al. 2007) and observational (Rüedi et al. 1992; Solanki et al. 1996) results.

During the evacuation, the dowflow velocities reach maximum values of $5-10 \mathrm{~km} \mathrm{~s}^{-1}$ at $\tau=1$. In the case of the largest feature, the downflow extends from the upper boundary of the simulation domain and becomes supersonic in the lower photosphere. The magnetic features formed have diameters of $0.1-0.2^{\prime \prime}$. In the case of the largest feature, a supersonic upflow develops approximately $200 \mathrm{~s}$ after the formation of the flux concentration. The upflow does not lead to a complete dispersal of the field, but the feature persists until 9 min later when it undergoes a fragmentation. The disappearance of the features in all three cases occurs when they meet opposite polarity features, between 3 and 20 min after their formation.

We also show what happens with the observables when the effects of smearing to observational spatial resolution is taken into account. An important result is that, in the case of small features, Hinode/SP would miss the bright point formation and, in some cases, also the high velocity downflows that develop in the process. On the other hand, the signatures of the evolution of large features are detectable even after the spatial smearing. We show that this case can be quantitatively compared with Hinode/SP observations (Nagata et al. 2008; Shimizu et al. 2008) and exhibits a very similar evolution. This suggests that the magnetic field intensification process in the MURaM simulations is a faithful description of the process taking place on the Sun. Furthermore, our study indicates that the analysis and interpretation of the observations in terms of the convective intensification process is well-founded.

Acknowledgements. Hinode is a Japanese mission developed and launched by ISAS/JAXA, with NAOJ as domestic partner and NASA and STFC (UK) as international partners. It is operated by these agencies in co-operation with ESA and NSC (Norway). We thank R. Cameron for valuable suggestions. This work was partially supported by WCU grant No. R:31-0016 funded by the Korean Ministry of Education, Science and Technology. This research has been partly supported by the Ministry of Science and Technological Development of the Republic of Serbia (Project No 146003 "Stellar and Solar Physics").

\section{References}

Bello González, N., Okunev, O., \& Kneer, F. 2008, A\&A, 490, L23

Bellot Rubio, L. R., Rodríguez Hidalgo, I., Collados, M., et al. 2001, ApJ, 560, 1010

Bercik, D. J., Nordlund, A., \& Stein, R. F. 2003, In Proceedings of SOHO 12 / GONG+ 2002. Local and global helioseismology: the present and future, ed. H. Sawaya-Lacoste, 201

Bonet, J. A., Márquez, I., Sánchez Almeida, J., et al. 2008, ApJ, 687, L131 Cheung, M. C. M., Schüssler, M., \& Moreno-Insertis, F. 2007, A\&A, 467, 703

Cheung, M. C. M., Schüssler, M., Tarbell, T. D., \& Title, A. M. 2008, ApJ, 687, 1373

Danilovic, S., Gandorfer, A., Lagg, A., et al. 2008, A\&A, 484, L17

Frutiger, C., Solanki, S. K., Fligge, M., \& Bruls, J. H. M. J. 2000, A\&A, 358, 1109

Gadun, A. S., Solanki, S. K., Sheminova, V. A., \& Ploner, S. R. O. 2001, Sol. Phys., 203, 1

Grossmann-Doerth, U., Schüssler, M., \& Steiner, O. 1998, A\&A, 337, 928

Jeong, J., \& Hussain, F. 1995, J. Fluid Mechanics, 285, 69

Khomenko, E., \& Collados, M. 2007, ApJ, 659, 1726

Kosugi, T., Matsuzaki, K., Sakao, T., et al. 2007, Sol. Phys., 243, 3

Lites, B. W., Elmore, D. F., \& Streander, K. V. 2001, in Advanced Solar Polarimetry - Theory, Observation, and Instrumentation, ed. M. Sigwarth, ASP Conf. Ser., 236, 33

Lites, B. W., Kubo, M., Socas-Navarro, H., et al. 2008, ApJ, 672, 1237

Muller, R. 1983, Sol. Phys., 85, 113

Muller, R., \& Roudier, T. 1992, Sol. Phys., 141, 27

Nagata, S., Tsuneta, S., Suematsu, Y., et al. 2008, ApJ, 677, L145

Nordlund, A. 1983, Solar and Stellar Magnetic Fields: Origins and Coronal Effects, 102, 79

Nordlund, A. 1986, Small Scale Magnetic Flux Concentrations in the Solar Photosphere, 83

Parker, E. N. 1963, ApJ, 138, 552

Parker, E. N. 1978, ApJ, 221, 368

Piskunov, N. E., Kupka, F., Ryabchikova, T. A., et al. 1995, A\&AS, 112, 525

Roudier, T., Malherbe, J. M., November, L., et al. 1997, A\&A, 320, 605

Rüedi, I., Solanki, S. K., Livingston, W., \& Stenflo, J. O. 1992, A\&A, 263, 323 Schüssler, M. 1990, in Solar Photosphere: Structure, Convection and Magnetic Fields, ed. J. O. Stenflo (Dordrecht: Kluwer), IAU Symp., 138, 161

Sheminova, V. A., \& Gadun, A. S. 2000, Astron. Rep., 44, 701

Shimizu, T., Lites, B. W., Katsukawa, Y., et al. 2008, ApJ, 680, 1467

Socas-Navarro, H., \& Manso Sainz, R. 2005, ApJ, 620, L71

Solanki, S. K., \& Bruls, J. H. M. J. 1994, A\&A, 286, 269

Solanki, S. K., Zufferey, D., Lin, H., et al. 1996, A\&A, 310, L33

Spruit, H. C., \& Zweibel, E. G. 1979, Sol. Phys., 62, 15

Stein, R. F., \& Nordlund, A. 1998, ApJ, 499, 914

Stein, R. F., \& Nordlund, Å. 2006, ApJ, 642, 1246

Steiner, O. 1999, Third Advances in Solar Physics Euroconference: Magnetic

Fields and Oscillations, ASP Conf. Ser., 184, 38

Takeuchi, A. 1999, ApJ, 522, 518

Thevenin, F. 1989, A\&AS, 77, 137

Tsuneta, S., Ichimoto, K., Katsukawa, Y., et al. 2008, Sol. Phys., 249, 167

Venkatakrishnan, P. 1986, Nature, 322, 156

Vögler, A. 2003, Ph.D. Thesis, University of Göttingen, Germany,

http://webdoc.sub.gwdg.de/diss/2004/voegler

Vögler, A., \& Schüssler, M. 2007, A\&A, 465, L43

Vögler, A., Shelyag, S., Schüssler, M., et al. 2005, A\&A, 429, 335

Webb, A. R., \& Roberts, B. 1978, Sol. Phys., 59, 249

Weiss, N. O. 1966, R. Soc. London Proc. Ser. A, 293, 310

de Wijn, A. G., Stenflo, J. O., Solanki, S. K., \& Tsuneta, S. 2008, Space Sci.

Rev., 144, 275 